\title{
The Prism of Elasticity in Rebound Effect Modelling: An Insight from the Freight Transport Sector
}

\author{
Franco Ruzzenenti 1,2 \\ 1 Energy and Sustainability Research Institute, University of Groningen, 9747 AG Groningen, \\ The Netherlands; f.ruzzenenti@rug.nl; Tel.: +31-5036-34-691 \\ 2 Advanced Systems Analysis, International Institute for Applied Systems Analysis, 2361 Laxenburg, Austria
}

Received: 17 July 2018; Accepted: 7 August 2018; Published: 13 August 2018

\begin{abstract}
If the rebound effect is to be considered a major obstacle to sustainable freight transport, then action and timely policy must be made in advance. This, however, requires a theoretical understanding of the nature of the rebound effect and an empirical grasp of its underlying mechanism. Elasticity is the centrepiece of current models on the rebound effect (or Jevons paradox). Although elasticity is a metric of indisputable usefulness for empirical purposes, it may be misleading when applied to the complex rebound effect. Drawing on the parallel case of the 'distance puzzle' in international economics, it will be shown how elasticity can be misinterpreted or how it can misdirect an investigation of the phenomenon by following a predetermined mindset. This particular bias is shown to widen in the long term and evolving systems in which the elasticity metric continues to output a constant number, eliciting a persistent effect. Drawing on previous research, an alternative approach to studying the rebound effect based on complex network theory and statistical mechanics of networks will be described. It will be shown how the interplay between spatial and non-spatial effects in freight transport networks can inform us about the evolution of the effect of distances on trade relationships, upon which a new metric for the rebound effect can be built.
\end{abstract}

Keywords: freight transport; rebound effect; Jevons paradox; gravity models; distance puzzle; network theory; statistical mechanics of networks; complexity

\section{Introduction}

In 1865, Stanley Jevons published his famous pamphlet 'The Coal Question', in which, for the first time in history, it was suggested that the consequences of energy efficiency might be very different from their intended, conservative goals. In fact, 'the very contrary is true': a more efficient technology delivers a more economical use of energy, thereby encouraging 'more and new applications' [1]. The Jevons paradox has surfaced several times throughout the history of energy and environmental studies and in such different circumstances and guises that in an editorial of a landmark special issue of Energy Policy, Lee Schipper referred to it as the 'Loch Ness monster' of energy efficiency [2]. The first occurrence was after the oil shocks of the 1970s in the guise of the 'Khazzoom-Brookes postulate', named as such after two economists who independently questioned the effectiveness of pursuing energy conservation by imposing efficiency mandates. Rejuvenated by a new climate change awareness, the second appearance came at the end of the century, bringing with it a new wave of energy efficiency announcements. This time it arrived in its definitive form, the rebound effect (RE), which remains the most common description of the paradoxical connection between energy efficiency and energy consumption. According to a widely used definition, the rebound effect is a 'behavioural or systemic response' to a new, more efficient technology, which can lead to partially or totally offsetting expected savings [3]. Despite it still being a controversial topic, often engendering exorbitant reactions, the rebound effect has attracted considerable scientific interest, as demonstrated by the volume of 
articles published in international journals every year, which increased more than six times in the last decade. Between 1998 and 2008, less than 10 articles per year addressing or mentioning the rebound (in abstract or text) could be found on Scopus, whereas, in the last five years, the number went from 50 to 60 articles, many of the them in the field of transport research.

The transport sector has always been a favoured subject for investigating the rebound effect. There are several reasons for this. It epitomizes the transformational power of energy efficiency more than any other field. It is often blessed with abundant data and offers a real world subject for conceptualizing RE [4]. This is why a car is very often used as a tangible example of the rebound effect. The thrill you experience if you buy a more efficient vehicle means you are likely to drive for leisure more often and choose driving a car in place of public transport. It also enables you to commute longer distances and choose a more distant workplace. The problem presented here is an emblematic case of behavioural response, but what is the analogous problem for freight transport? There is probably none to be found in cargo or railway freight, where behavioural changes are difficult to envisage, and few in the case of road freight. More efficient trucks could, feasibly, encourage more aggressive driving behaviour, but this is arguably only a secondary factor in road freight transport energy consumption. Rather than a behaviour response, energy efficiency has always had seen a more profound response in transport costs and what those are inimitably intertwined with: the spatial and time distribution of the value chain, both up-stream (supply chain) and down-stream (distribution and retail). Market integration of factors and goods is just one of the positive, unintended consequences of more efficient transportation that has always had an arguably deep impact on energy demand [5]. Indeed, in this field, the balance between behavioural and systemic responses seems to lean towards the latter and makes the analysis of the rebound effect far more complex and with much wider implications. The transport-intensive economy which arose after Fordism was initially supported by the road transport sector (in the words of Baldwin [6] it was the first 'unbundling') and later by the cargo and aviation sector (the second 'unbundling') - it is not possible to disentangle the evolution of global value chains from that of transport costs whose energy efficiency is a non-marginal component [7]. In this article, after briefly reviewing the literature about the rebound effect in freight transport (method and findings), what could be considered the prism of almost all current models on RE will be considered - the energy service to energy efficiency elasticity-to show how a rebound effect metric based on elasticity can be very misleading in the long term when there are structural effects in the system. To do this, it will be drawn on the experience of gravity theory in trade economics, where the inability of models to capture declining transport costs sparked a long-standing debate (the 'distance puzzle'). A completely different approach will be proposed based on (complex) network theory and statistical network mechanics to model the rebound effect in freight transport, followed by contemplation of how and where future research should proceed.

\section{Rebound Effect in Freight Transport: The Role of Elasticity}

Although the number of studies on private mobility is much higher, the increasing interest over the past decade on the rebound effect in freight transport (mostly road freight) has partially closed the gap. Table 1 offers a snapshot of the literature. The work of Walnum et al. [8] provides a more complete and in-depth review of the subject and, despite being current only to 2014, frames the issue clearly in the context of rebound effect studies and sustainability in transportation. The percentage shown in the last column of Table 1 indicates how much of the expected savings rebounds within a specific time-span. A $100 \%$ rebound value means that all expected savings are absorbed by increased demand. A value greater than $100 \%$ indicates that consumption grew higher than before the new technology was introduced. Table 1 reports authors, case study (country and time span) and the metric used. With a few exceptions, most are econometric regressions $[9,10]$. The variables used to assess the rebound effect are: energy service (tonne-kilometres or vehicle-kilometres) or energy consumption (diesel) versus energy efficiency (generally fuel economy, per vehicle or tonne-kilometres) or energy prices (taken as a proxy of energy efficiency). When possible, regressions are performed on all the 
variables above, plus some additional explanatory variables (such as economic output, population, load factors, etc).

Table 1. Rebound effect (RE) in road freight transport, estimates review: the range of values refer to different regions, countries or type of vehicles; values in parenthesis refer to short-term estimates.

\begin{tabular}{cccc}
\hline Study & Case Study & Metric & Estimate \\
\hline Anson and Turner [9] & Scotland, 1999 & Service, efficiency & $36-39 \%$ \\
Matos and Silva [11] & Portugal, 1987-2006 & Service, efficiency & $24 \%$ \\
Borger and Mulalic [12] & Denmark, 1980-2007 & Service, efficiency & $17 \%(10 \%)$ \\
Wang and Lu [13] & China, 1999-2011 & Service, price & $52-84 \%$ \\
Leard et al. [14] & USA, 1977-2002 & Service, price & $9-30 \%$ \\
Winebrake et al. [15,16] & USA, 1997-2006 & Service, price and efficiency & $0 \%$ \\
Llorca and Jamasb [10] & EU(15), 1992-2012 & Energy, price & $0-68 \%$ * \\
Sorrell and Stapleton [17] & UK, 1970-2014 & Service, efficiency and price & $21-137 \%$ \\
\hline
\end{tabular}

$\left.{ }^{*}\right)$ indicates a $0-100 \%$ RE range imposed by the model.

The most accepted measure [18] of the rebound effect is the elasticity of demand for energy services $S$ with respect to energy efficiency $\epsilon$. However, when (physical) efficiency data is lacking, the elasticity of energy services with respect to energy price $p$, namely $\eta_{\epsilon}(S)$ and $\eta_{p}(S)$, is often used. When there is no data on energy services, the following relation holds between the elasticity of energy consumption $E$ and energy services $S$ over efficiency (price): $\eta_{\epsilon(p)}(E)=\eta_{\epsilon(p)}(S)-1$ [18]. Regardless of the approach used, elasticity has become a popular metric to assess the rebound effect, though there are some exceptions (for example, the model by [10]). In most cases (including beyond the domain of transportation), it acts as a prism by which we look at the paradoxical phenomenon of greater energy efficiency leading to higher energy consumption.

The present article does not discuss the limitations of other approaches, nor provide a comprehensive survey of those few that do not use elasticity as a metric for the rebound effect (several studies have used Life Cycle Assesment(LCA), a good review is given by Font Vivanco [19] and there is also the work of Freeman et al. who estimate RE using system dynamics modelling [20]. Others have used decomposition analysis, which is ultimately still based on elasticity). Instead, this article aims to show how elasticity has ultimately shaped our perception of the rebound effect. Indeed, elasticity has taken a paradox (a thing existing in the domain of words) and turned it into a number (a thing we can measure and count). The reasons for its success are not only empirical but also axiomatic. The concept of elasticity combines well with the rebound effect because both indicate a state of quietness or balance, which is suggestive of a particular level of energy savings without RE. This is an important analogy because, once we have decided to measure the rebound effect, we must first be able to set the expected consumption level without the rebound effect. Elasticity provides us with a suitable solution for this problem with the value 0 (perfectly inelastic). This level need only be an estimate. The elasticity concept presents the idea that RE is inherent to the system, like a durable property, in the same way certain materials are more or less flexible. This is consistent with the idea that elasticity is immanent and persistent. Nearly all rebound effect studies assume that this does not change over time. In some cases, the estimations given for the rebound effect span three decades or more, but can we reasonably assume that the 'behavioural or systemic response' of the system remains the same over such a long period of time? Are behaviours today the same as they were in the 1970s? Is the transport system the same? Does it use the same means and infrastructure? The next chapter will show how similar questions have puzzled trade economics scholars for years and specifically in terms of elasticity and longer timescales.

\section{The 'Distance Puzzle'}

In 1954, using a remarkably simple and elegant idea, the economist Walter Isard proposed a model emulating gravity theory to predict trade flows (Some give credit to Pöyhönen [21] and others to Tinbergen [22] for the introduction of gravity models in economics, but this dispute is beyond the 
scope of the present article). The concept follows that the amount of trade between two countries should be directly proportional to a measure of size (typically gross domestic product (GDP)) and inversely proportional to distance [23]. The simplest formulation of this relationship is:

$$
w_{i j}=G \frac{x_{i} x_{j}}{d_{i j}^{\gamma}}
$$

where $w_{i j}$ is the flow between country $i$ and country $j, G$ is an empirical constant (the 'gravitational constant'), $x$ is the GDP and $\gamma$ is the power factor of distance $d$ (a quadratic law, for mass-mass interaction).

Gravity models (GM) are a whole family of spatial interaction models rather than a single model. Wilson's pioneering work provides a clear categorization of gravity models according to parameter specifications: production, attraction and attraction-production constrained models. In the first case, the model is parameterized according to observed flows, in the second to the observed mass term and in the last case the model is constrained to both. Remarkably, Wilson also proposes a maximizing entrop gravity model where the general function is replaced by an exponential function and the resulting value indicates the expected flow given the observed constraints. The functional form, obtained by maximizing the log (likelihood), is the same as the exponential random graph models, but in the case proposed here, expected values are intended as a null model rather than predictions of future flows. Furthermore, Wilson's approach is dyadic (bilateral) and does not consider the topology of the whole transport system as we do in our previous works. Nevertheless, the similarity is striking and Wilson's intuition remarkable [24]. Gravity models proved to be good predictors of trade volumes but exhibited major shortcomings [25]. As a result, they have seen a long, uneven success and found as many advocates as detractors prompting as much research to improve them as to overcome them. For example, a limitation is their inability to predict zero-flows in a world where not all countries are linked by trading relationships (i.e., the network of trade is not fully connected). A second major flaw is that GM predict perfectly symmetrical flows between each pair of countries: this is also empirically untrue [26]. Mutual relationships are indeed relevant. However, bilateral trade is never perfectly balanced, nor is the trade matrix perfectly symmetrical [27]. Both findings are not surprising given that gravity force presents a rotational symmetry, i.e., particles along the same radius experience the same force. In other words, in Newton's third law (action-reaction), two particles at a given distance experience the same force (obviously, the same relationship does not apply to the volume of trade between two countries). In essence, the problem is that GM assume trade occurs in a homogeneous space, but, in reality, trade occurs in an oriented, structured space [28].

Nevertheless, scholars have been mostly bewildered by the fact that empirical estimation of 'distance coefficients' (measuring the effect of distance on trade) were practically constant over time [29]. In a widely cited book, Frances Cairncross [30], senior fellow at UCLA (University of California, Los Angeles), proclaimed the 'death of distances' as a result of advancements in communication and transport technologies. However, as remarked by Lin and Sim, 'while the death of distance seems sensible in light of globalization, the task of establishing this empirically has proven to be challenging' [31]. This 'task' has triggered a vast and varied scientific endeavour to find declining distance coefficients in time, mostly in the field of economics whose reach does not extend to this article (nonetheless, it is worth recalling three works which notably address the 'distance puzzle' by modifying its most crucial hypothesis: the homogeneity of space. The first article describes a model to study the spatial homogeneity in trade by assessing the autocorrelation in trade volumes with Hurst exponent [28]. The second introduced fractality to interpret the distance coefficients [32] and the third introduced topological constraints to a radiation model [33]). Hence, where trade economists were desperately searching for a pattern of change, scholars interested in RE were looking for immutability. The goal of these was to show that elasticity between trade and distance was changing over time, whereas the others aimed to measure constant elasticity (of transport service to energy efficiency). Both were motivated by an unspoken vision of the system which in one case struggled to correspond with the paradigm expressed by model and metric, and in the other 
case aligned perfectly with the model. This was a model whose idea of hypostasis was reassuring or, in other words, had an underlying substance: a fundamental, immutable reality behind it. The concept of elasticity in economics resembles that in materials sciences where the relationship between stress $\delta$ and strain $\epsilon$ (or deformation) is proportional to a constant $E$ :

$$
\delta=E \epsilon
$$

Deformations $(\epsilon)$ generated by external forces $(\delta)$ can vary according to boundary conditions (i.e., temperature), but are essentially pre-determined by some fundamental structural properties of the molecules' matrix measured by E. In solid-state physics, the molecular lattice changes size and shape when forces are applied (which means that the energy of a system increases) and when these cease the lattice returns to its original lower energy state. As for the 'lower energy state', in the RE model, this is analogous with the sought-after 'state of normality' which is essential to establish the hypothetical energy consumption in which energy efficiency has expressed all its potential. However, the structure of interactions in social and economic systems is more like a complex network than a regular matrix and trade (or freight transport) is not an exception [34]. This is not a trivial question because the dynamics of processes (such as shock propagation or epidemic phenomena) on complex networks can be remarkably different from that on a lattice or lattice-like systems [35]. The space of interactions in a complex network is not homogeneous and the incumbent structure (topology) of agents' interactions shape the speed, frequency and intensity [28,32]. Complex network theory proved to be a highly informative approach to understand trade, its connection with economy [36] and how this evolved over time [37]. Can network theory help investigate RE in freight transport using an approach which is evolutionary and takes into account the complexity of the system? In the next section, an attempt recently taken in this direction [38] will be illustrated.

\section{Modelling Rebound Effect with Network Theory}

Network theory primarily focuses on the study of interactions, in contrast to most scientific approaches which are concerned with the study of their constituents. It is also a holistic approach because it studies the structure emerging from the entire organization of nodes. Specifically, network theory focuses on how the combination of many local interactions, generally formed following a decentralized and non-engineered process, can give rise to unexpected structures at a global level. In turn, these non-designed global (macroscopic) properties can affect the properties of individual nodes or edges and, consequently, the local (microscopic) structure. Interactions between intermediate (mesoscopic) levels are also possible, for instance when the entities under study are so-called communities, i.e., sets of vertices more densely connected internally than with the rest of the network.

In recent years, the use of complex network theory in the field of transport studies has gained momentum mostly, but not exclusively, in air transport [39]. Two articles that provide an extensive and detailed review of the subject are [40,41]. A recent attempt by Calatayud et al. used a network (and multi-network) approach to study global trade as a transport network, though it must be said that this study lacks analysis of any real spatial input (that is, its spatial embedding) as it only concerns topological properties [42]. Network theory has also gained the attention of supply chain analysis scholars as a way of overcoming the established linear and static view of production processes using 'complex adaptive systems' [43]. Similar to the idea presented here, but at a lower scale of analysis, supply chains are approached as evolving networks exchanging 'materials and information' [44]. Few studies, however, take a strictly quantitative approach to describe the structure and properties of such networks [45]. Interestingly, according to a wide and accurate review dated 2013, none of the 126 articles assessed consider supply chain networks as spatial networks, where spatial effects (or transports) would be a factor shaping their topology [46]. Even when logistics are considered in 
the analysis, they appear to be adaptive to the network structure rather than the opposite. The spatial fix of the network's nodes is given.

Needless to say, to establish the connection between energy efficiency and a transport network, the spatial embedding (also known as geographical or Euclidean embedding) of the network and the spatial information it provides is of paramount importance. It is worth noting that a network is an abstract object described by a matrix whose entries are specified by the existence (or the magnitude, in the case of weighted rather than binary graphs) of a link between two elements of a set. Mathematically, the embedding of the network in space is irrelevant. In principle, different spatial configurations are just different arbitrary representations of the same graph. However, if we are looking for explanations of the structure of a particular network or predictions of its future evolution, then it might well turn out that the spatial information is relevant. Networks that are subject to strong spatial constraints generally display a high degree of regularity and so are more predictable (depending on the spatial information) than other graphs. For example, in a lattice, each atom has the same number of connections (degree). Conversely, complex networks show a much more complex architecture with a degree distribution following a (non-regular) power law. In a complex transport network, the transport system embodies the spatial information concerning the topological configuration which we expect to be affected by the efficiency of transport. In conclusion, we have on the one hand the topological information delivered by the structure of the complex network (for example, the trade network), and on the other hand the spatial information delivered by the transport system (i.e., the spatial network), and we want to combine the two to obtain information about the mutual influence.

Following previous work on spatial networks [47], it will be now shown how to gauge spatial effects on complex networks aimed at developing a spatial embeddin metric of a network upon which, as will become clearer later, it is possible to estimate RE. The trade network of 27 European countries (EU27) will be used as a case study. The two dimensions of the analysis, topological and spatial, are condensed into two matrices, the matrix $\mathbf{A}$ of trade relationships and the matrix $\mathbf{D}$ of distances (Figure 1).

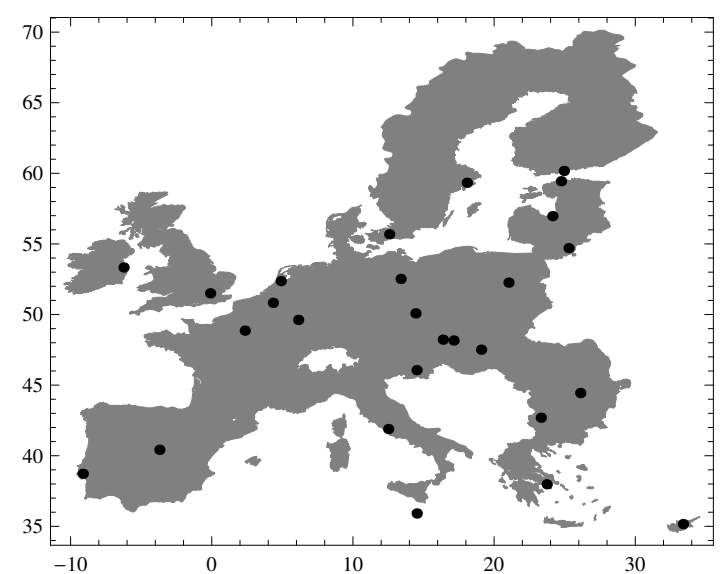

(a) matrix $\mathbf{A}$

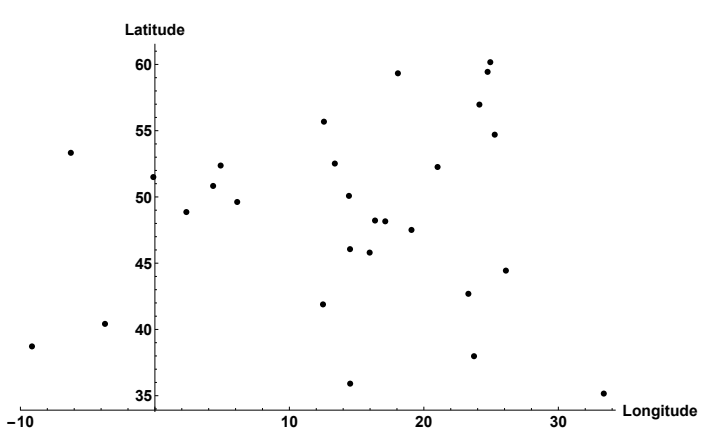

(b) matrix D

Figure 1. The cross-border transport network of Europe condensed into its (a) topological dimensions (bilateral trading relationships, whose entries are trade flows in mass units (tonne)) and (b) spatial dimensions (binary distances between capitals, whose entries are geodesic distances measured on longitudes and latitudes (deg)).

The simplest, linear choice for a measure that exploits the entire topological and spatial information provided by the network is:

$$
F \equiv \sum_{i=1}^{n} \sum_{j \neq i} a_{i j} d_{i j}
$$


where $a$ and $d$ are the entries of the binary matrix $\mathbf{A}$ (a matrix with entries 0 or 1 if a link exists between tow nodes) and $\mathbf{D}$ respectively and $n$ is the number of nodes of the network ( 27 in our case). The second step is normalization because the value $F$ expressed in Equation (3) is not a suitable measure for comparing different networks or the evolution of a single network in time as it is size-dependent. Networks can grow in terms of number of links (edges) or nodes (vertexes) and in the latter case also the embedding space. Therefore, we consider a slightly more complicated definition for the single purpose of having a normalized quantity ranging between 0 and 1 :

$$
f \equiv \frac{\sum_{i} \sum_{j \neq i} a_{i j} d_{i j}-F_{\min }}{F_{\max }-F_{\min }}
$$

where $F_{\min }$ and $F_{\max }$ are the minimum and maximum values that $F$ can take, given the distance matrix $\mathbf{D}$ and the total number of links $L$. The vector $V^{\uparrow}=\left(d_{1}^{\uparrow}, \ldots, d_{n}^{\uparrow}, \ldots, d_{N(N-1)}^{\uparrow}\right)$ denotes the list of all (off-diagonal) distances ordered from the smallest to the largest where $d_{n}^{\uparrow} \leq d_{n+1}^{\uparrow}$ and symmetrically the vector $V^{\downarrow}=\left(d_{1}^{\downarrow}, \ldots, d_{n}^{\downarrow}, \ldots, d_{N(N-1)}^{\downarrow}\right)$, considers from the largest to the smallest distances, where $d_{n}^{\downarrow} \geq d_{n+1}^{\downarrow}$. Hence, in terms of the two lists $V^{\uparrow}$ and $V^{\downarrow}$, the maximum and minimum values for $F$ read $F_{\min }=\sum_{n=1}^{L} d_{n}^{\uparrow}$ and $F_{\max }=\sum_{n=1}^{L} d_{n}^{\downarrow}$. It is worth noting that the matrix $A$ is non-relevant to the computation of $F_{\min (\max )}$ as it only depends on the existing number of links $L$ and it is not influenced by the observed topology. The value ranges between 0 and 1 . The former extreme $(f=0)$ represents the case where the $L$ links are placed among the closest pairs of nodes (the maximum shrunk network). The latter extreme $(f=1)$ instead represents the case where the $L$ links are placed among the most distant pairs of nodes (the maximum stretched network). In our EU27 trading network example, these two extremes are shown in Figure 2a,b respectively, where, for visualization purposes, we have actually chosen a value of $L$ equal to $n=27$, much less than the real value (which would fill the plot with links). This would have been the case, for instance, if in the original network each node had exactly one outgoing link. Networks between the two extremes would have a value $0<f<1$. As rendered intuitively by the figures, a larger value of $f$ implies a more pronounced filling of the available space. Therefore, we denote $f$ as the (spatial) filling of the network represented by the matrix $\mathbf{A}$.

So far, we have considered the binary network, but an extension of $f$ to the weighted network, which is more suitable for assessing transport flows, reads as follows:

$$
f_{w} \equiv \frac{\sum_{i} \sum_{j \neq i} w_{i j} d_{i j}-F_{\min }}{F_{\max }-F_{\min }},
$$

where $w$ is the entry of the weighted matrix and $F_{\min }$ and $F_{\max }$ are chosen as the two extreme values that $F$ can take in a network with the same total weight $W\left(\sum_{i} \sum_{j \neq i} w_{i j}\right)$ as the original network, i.e., $F_{\text {min }}=W d_{1}^{\uparrow}$ and $F_{\max }=W d_{1}^{\downarrow}\left(d_{1}^{\uparrow}\right.$ and $d_{1}^{\downarrow}$ are the smallest and largest distance between vertices, respectively). The concept here is that from the binary to the weighted representation, unitary links can cumulate between dyads, forming a (weighted) flow as a discrete summation of unitary links (similarly, particles that follow the Bose-Einstein distribution can occupy the same energy level). Therefore, the maximally shrunk(expanded) configuration is when all links cumulate between the closets(farthest) couple of nodes, that is, when the total weight is placed on the shortest(longest) distance. A different normalization could be done preserving the local weight distribution. In this case, the $F_{\min (\max )}$ would result as the summation of the total strength of the node $i$ times the distance with the closest (farthest) $j$, for al the $i$ nodes. This would change the weighting factor, but leave the trend unaffected, which is the focus of the present analysis. This measure, like its binary equivalent, will vary between 0 and 1 (though the visual representation is more complex here) and informs us about the spatial embedding of the network: the lower the value, the higher the embedding, that is, distances are more important in determining the network's interactions (topology). Can we use the weighted filling (Equation (5)) 
to assess the rebound effect? This measure indeed gives us an indication of the role of distance in shaping the network topology, but we know that topology is never entirely, if only ever marginally, explained by its spatial constraints [48]. We expect, for example, the Netherlands to trade abundantly with Germany not just because of their geographic proximity, but because Germany is a hub in the European trading network. Therefore, to carve out the purely spatial information found in trading relationships, we need to disentangle spatial and non-spatial (topological effects) using a null model based on exponential random graph theory and maximum entropy graph ensembles [49]. A maximum entropy graph ensemble is, in essence, a sample of randomized networks that conserve some desired properties similar to the network under investigation, say the in and out degree sequence (or in and out strength sequence for weighted networks). The functional form of maximum entropy ensemble can be solved analytically or performed by means of an iterative process. In this ensemble, we can assess the expected (average) value of the investigated measure (for example, the filling) in order to gauge if this is trivially explained by the constraints we imposed on the ensemble (say, the sequence of imports and exports observed in the network). We are thus able to implement the expected filling value of the spatial filling and control for non-spatial effects embodied in a null model via the filtered filling $\phi$ :

$$
\phi \equiv \frac{f-\langle f\rangle}{1-\langle f\rangle}
$$

where $\langle f\rangle$ is now obtained by replacing $w_{i j}$ with $\left\langle w_{i j}\right\rangle$ (the flows generated by the null model) in Equation (5). Positive (negative) values of $\phi$ indicate a network which is more stretched (shrunk) than expected by the null model (hence, by imposing some topological constraints). Notably, for a space-independent network, $\phi=0$ sets the level of spatial neutrality, that is, the level at which the spatial embedding of the network is fully (and trivially) explained by its topological properties. The network is therefore indifferent to distance, meaning that it is neither positively nor negatively affected by distance. Arguably, a more efficient technology will reduce the weight of distance in trade relationships and drive the system towards spatial neutrality. In Figure 3, we show how the decade-long trend of filtered spatial filling $\phi$ of Europe compared to two measures of energy efficiency [38]. Amid a fast increase in energy efficiency, as marked by both the energy intensity of freight transports (toe/tkm) and fuel economy of trucks $(\mathrm{L} / \mathrm{km})$, the filtered filling becomes more negative, indicating that the system increased its spatial embedding (given its topology). In other words, distances have become more binding, even if only marginally (a variation of the order of $10^{-2}$ ). Overall, the value of $\phi$ of Europe shows us that the European transport network is very close to spatial neutrality and thus is not significantly affected by efficiency variations and we should not see a rebound effect. This is peculiar to Europe. Other spatial networks generally show a marked negative value, decreasing with time (showing that distances become less binding). For example, the world as a whole displays a negative $\phi$ of -0.2 [38]. This result is in line with that obtained by [10] who also found a close-to-zero rebound effect for Europe during the same period. Nevertheless, broadening the time scope of the analysis suggests that this result might only be a true for Europe in the last few decades.

Figure $3 \mathrm{~b}$ shows the long-term trend of spatial filling for Europe measured in monetary units instead of mass units. The long-term trend of the filling value shows that the European trade network has undergone two marked phases: an initial phase of spatial contraction which ended in the mid-1970s, and a following phase of spatial expansion which ended in the mid-1990s. It is plausible that, after this second wave of spatial expansion, Europe entered a stable, spatially-neutral phase as portrayed by the analysis of the last decades (Figure 3a). Consequently, RE was more marked and effective between the 1970s and the 1990s than in the last two decades. However, this is just a tentative analysis. More research is needed, supported by reliable and fine-grained data on mass flows and efficiency in freight transports. 


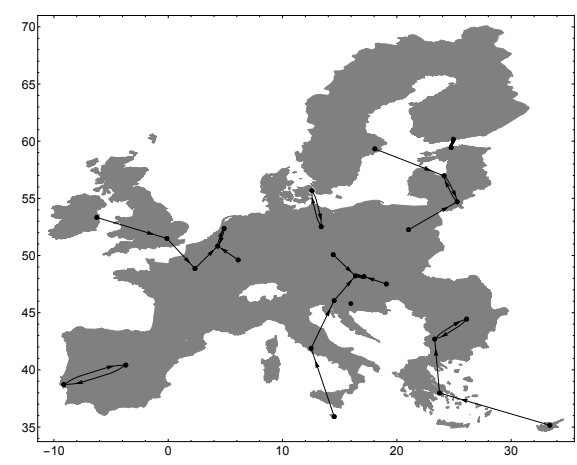

(a)

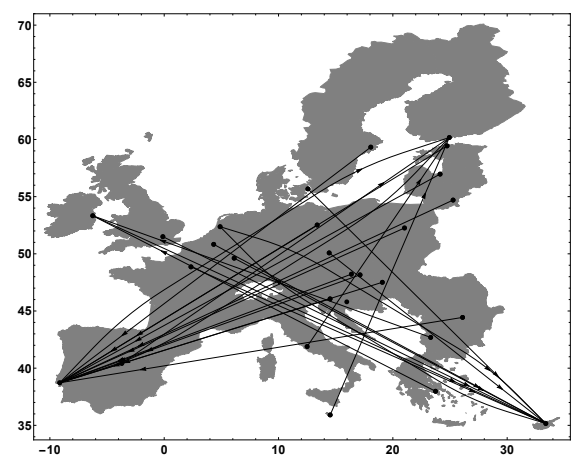

(b)

Figure 2. The two extreme degrees of spatial embeddedness (filling) for the binary trading network of Europe. (a) Maximum shrunk configuration for the EU27 trading network with a constraint on the number of links: $f=0(N=27, L=27$; (b) Maximum stretched configuration for the EU27 trading network with a constraint on the number of links: $f=1(N=27, L=27)$.

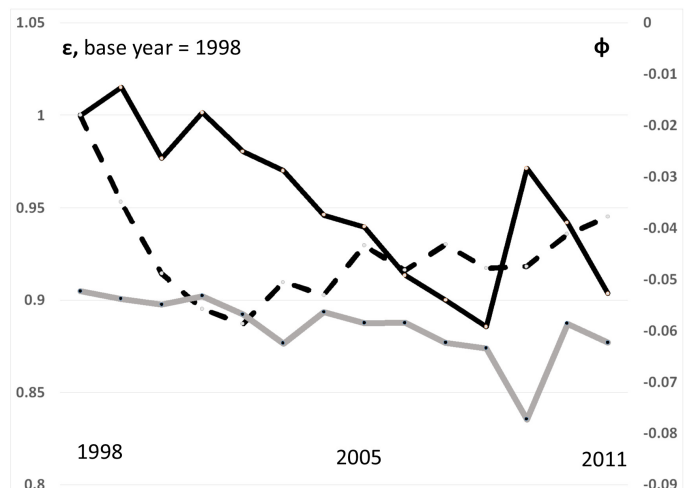

(a) Filtered filling and efficiencies

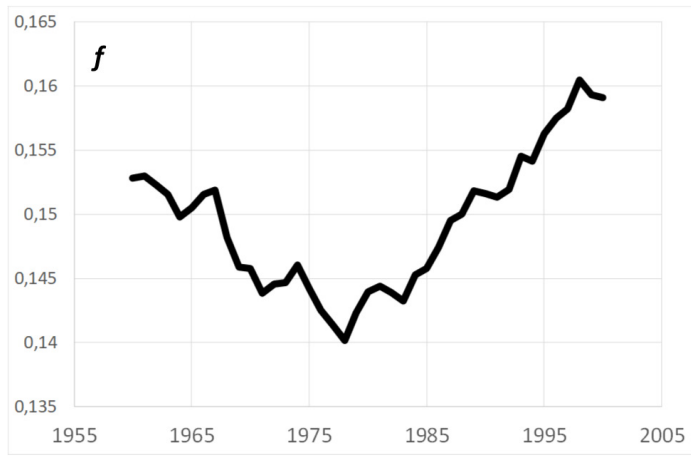

(b) Trend of spatial filling

Figure 3. Trends in European (EU27) spatial embedding: (a) Filtered filling $\phi_{w}$ in (gray line); fuel economy (dashed black line) and energy intensity (black line). Data source: CEPII www.cepii. fr/CEPII/en/bdd_modele/bdd.asp and Odysee http:/ / www.indicators.odyssee-mure.eu/energyefficiency-database.html. (b) Unfiltered filling $f_{w}$ (Equation (4) assessed on trade relationships in monetary units (black line). Data source: Gleditsch http:/ /journals.sagepub.com/doi/abs/10.1177/ 0022002702046005006.

Except in the last two decades where results are difficult to interpret, the model incontrovertibly shows that, after the late 1970s, spatial embedding of the European trade network fell dramatically (and the network expanded). The oil shocks triggered a drive for higher efficiency across all sectors 
of the economy but foremost the automotive sector [50]. As a result, road freight transport became the most economical mode of transport for goods and trucks became the conduit for the onset of the new transport intensive, post-Fordian economy. Between 1970 and 1995, the tKm of the road freight transport sector in Europe (EU15) grew more than 130\% compared to a growth rate of GDP and industrial production of 53\% and 56\%, respectively. In Italy, between 1973 and 1990, the traffic density of goods vehicles grew $132 \%$ (that of semi-trailers alone accounted for a whopping $172 \%$ ) as opposed to a growth rate of $25 \%$ for all types of road vehicles [5]. The environmental burden of the transition towards a transport intensive economy (a transition that has never reversed, but only attenuated or transferred to greater spatial scales) is twofold: local air quality deteriorated and global greenhouse gas emissions rose. Measures to counteract this burden are doomed by the rise of the diesel engine, whose unquestionable dominance appears now to be unchallenged. If energy efficiency triggered this transition, can it continue to be considered a potential solution or obstacle for achieving more sustainable transport?

\section{Conclusions}

Can rebound effects explain why sustainable mobility has not been achieved? This was the difficult question Walnum, Aall and Løkke addressed in an article published in this journal four years ago [8]. Far from giving a definitive answer, the authors came to the conclusion that under 'certain circumstances', energy efficiency improvements could lead to an overall increase in transport volume and completely offset energy savings, concluding with a measure of resignation that the rebound effect, 'will be evident as long as the economy keeps growing'. If RE is a serious threat to any energy-efficiency based strategy aiming for a more sustainable freight transport system, understanding its phenomenology is of paramount importance. Understanding the phenomenology means being able to decipher its true, complex nature and ideally its causes with a clearer insight into the scope, timescale and all its tangled interactions. Reducing RE to a number might be of practical use for gauging, for example, short-term, counteractive measures or estimating immediate response to a new, more efficient process, but, unfortunately, it does not add much to our fundamental comprehension. On the contrary, it may persuade us that the true nature of RE is a number. A number, and even more so an unequivocal one, can be reassuring, but it can also be soporific, especially when it is applied as a verdict or definitive response to a complex subject. With very few exceptions, elasticity is the prism through which we currently look at RE, regardless of the model used. In this article, an attempt was made to define elasticity as a metric for RE. Epistemological scrutiny showed that elasticity shapes our perception of RE by eliciting the idea that this is an inherent and permanent phenomenon rather than an evolutionary process. While studies on RE aim to measure the constant elasticity of energy service to energy efficiency, trade economics scholars are puzzled by the unchanged elasticity of trade to distance. Sometimes, we are misled by our expectations, even more so if the model we use is reassuring. Studies on RE should explore new avenues of research and join with different disciplines to gain new, different perspectives on the topic (For further reading on new approaches and models on RE: https:/ / www.frontiersin.org/research-topics/6598/the-rebound-effect-and-thejevons-paradox-beyond-the-conventional-wisdom). In this article, network theory was proposed as a new method for approaching the rebound effect in transport and the use of spatial (filtered) filling as an alternative metric to elasticity. Structural change and complexity are endemic to the network theory paradigm and the large amount of data the network provides annually (702 data points for the EU27, which is one order of magnitude greater than the annual data used for regression) allows us to perform sound statistical analysis every year and observe the evolution of the system as time elapses. Therefore, by inspecting the topology of interactions and their relation to spatial constraints (distances), a complexity and evolutionary perspective of the system is achieved. With spatial filling, we can observe spatial embedding of the network (contraction or expansion) and with filtered filling we can set the level of spatial neutrality (indifference) and the extent to which the observed network extends. By comparing these two measures with the evolution of energy efficiency, we have an indication of 
how the rebound effect unfolds over time, that is, how the spatial embedding responds to increased transport efficiency. Lastly, a question which should be addressed is whether a network approach to RE can be extended to sectors other than transportation. Our approach takes advantage of the concept of spatial embedding [38] and studies the interplay between the adjacency matrix and the distance matrix (Figure 1). A straightforward extension of this conceptualization to non-metric distances (non-spatial networks), although appealing, is unconvincing. It is also difficult to apply this framework to private mobility where distance is only one factor in decision-making. However, with increasing attention and research on Jevons paradox, it is expected that more scholars and scientists will follow the path of complex network theory, leading to new models and metrics, and new insights and puzzles.

Funding: This research received no external funding.

Acknowledgments: I would like to thanks Mir Mohammadi Kooshknow for the insightful comments and attend revision of the paper and two unknown reviewers for helping me in discovering a new ambit of knowledge of interest and enhancing my, indeed poor, dissemination skill.

Conflicts of Interest: The author declares no conflict of interest.

\section{Abbreviations}

The following abbreviations are used in this manuscript:

$\begin{array}{ll}\text { RE } & \text { Rebound Effect } \\ \text { GDP } & \text { Gross Domestic Product } \\ \text { GM } & \text { Gravity Models } \\ \text { LCA } & \text { Life Cycle Assessment } \\ \text { EU27 } & \text { European Union, 27 countries. } \\ \text { toe } & \text { Tonnes of Oil Equivalent } \\ \text { tKm } & \text { Tonnes Kilometres } \\ \eta & \text { elasticity } \\ \text { E } & \text { energy } \\ \text { S } & \text { energy service } \\ \epsilon & \text { energy efficiency } \\ p & \text { energy prices }\end{array}$

\section{References}

1. Madureira, N.L. The anxiety of abundance: William Stanley Jevons and coal scarcity in the nineteenth century. Environ. Hist. 2012, 18, 395-421. [CrossRef]

2. Schipper, L. On the rebound: The interaction of energy efficiency, energy use and economic activity. An introduction. Energy Policy 2000, 28, 351-353.

3. Maxwell, D.; Owen, P.; McAndrew, L.; Muehmel, K.; Neubauer, A. Addressing the Rebound Effect, a Report for the European Commission DG Environment; European Commission DG ENV: Bruxelles, Belgium, 2011.

4. Greening, L.A.; Greene, D.L.; Difiglio, C. Energy efficiency and consumption-The rebound effect-A survey. Energy Policy 2000, 28, 389-401. [CrossRef]

5. Ruzzenenti, F.; Basosi, R. The rebound effect: An evolutionary perspective. Ecol. Econ. 2008, 67, 526-537. [CrossRef]

6. Baldwin, R. Trade and Industrialisation after Globalisation's 2nd Unbundling: How Building and Joining a Supply Chain Are Different and Why It Matters (No. W17716); National Bureau of Economic Research: Cambridge, MA, USA, 2011.

7. Picciolo, F.; Papandreou, A.; Hubacek, K.; Ruzzenenti, F. How crude oil prices shape the global division of labor. Appl. Energy 2017, 189, 753-761. [CrossRef]

8. Walnum, H.J.; Aall, C.; Løkke, S. Can rebound effects explain why sustainable mobility has not been achieved? Sustainability 2014, 6, 9510-9537. [CrossRef]

9. Anson, S.; Turner, K. Rebound and disinvestment effects in refined oil consumption and supply resulting from an increase in energy efficiency in the Scottish commercial transport sector. Energy Policy 2009, 37, 3608-3620. [CrossRef] 
10. Llorca, M.; Jamasb, T. Energy efficiency and rebound effect in European road freight transport. Transp. Res. Part A Policy Pract. 2017, 101, 98-110. [CrossRef]

11. Matos, F.J.; Silva, F.J. The rebound effect on road freight transport: Empirical evidence from Portugal. Energy Policy 2011, 39, 2833-2841. [CrossRef]

12. De Borger, B.; Mulalic, I. The determinants of fuel use in the trucking industry-Volume, fleet characteristics and the rebound effect. Transp. Policy 2012, 24, 284-295. [CrossRef]

13. Wang, Z.; Lu, M. An empirical study of direct rebound effect for road freight transport in China. Appl. Energy 2014, 133, 274-281. [CrossRef]

14. Leard, B.; Linn, J.; McConnell, V.; Raich, W. Fuel Costs, Economic Activity, and the Rebound Effect for Heavy-Duty Trucks; Discussion Paper; Resources for the Future: Washington, DC, USA, 2015.

15. Winebrake, J.J.; Green, E.H.; Comer, B.; Corbett, J.J.; Froman, S. Estimating the direct rebound effect for on-road freight transportation. Energy Policy 2012, 48, 252-259. [CrossRef]

16. Winebrake, J.J.; Green, E.H.; Comer, B.; Li, C.; Froman, S.; Shelby, M. Fuel price elasticities for single-unit truck operations in the United States. Transp. Res. Part D Transp. Environ. 2015, 38, 178-187. [CrossRef]

17. Sorrell, S.; Stapleton, L. Rebound effects in UK road freight transport. Transp. Res. Part D Transp. Environ. 2018, 63, 156-174. [CrossRef]

18. Sorrell, S.; Dimitropoulos, J. The rebound effect: Microeconomic definitions, limitations and extensions. Ecol. Econ. 2008, 65, 636-649. [CrossRef]

19. Vivanco, D.F.; van der Voet, E. The rebound effect through industrial ecology's eyes: A review of LCA-based studies. Int. J. Life Cycle Assess. 2014, 19, 1933-1947. [CrossRef]

20. Freeman, R.; Yearworth, M.; Preist, C. Revisiting Jevons' paradox with system dynamics: Systemic causes and potential cures. J. Ind. Ecol. 2016, 20, 341-353. [CrossRef]

21. Pöyhönen, P. A tentative model for the volume of trade between countries. Weltwirtschaftliches Arch. 1963, 90, 93-100.

22. Tinbergen, J.; Hekscher, A. Shaping the World Economy: Suggestions for an International Economic Policy; Twentieth Century Fund: New York, NY, USA, 1962.

23. Walter, I. Location Theory and Trade Theory: Short-Run Analysis. Q. J. Econ. 1954, 68, 305-320. [CrossRef]

24. Wilson, A.G. A family of spatial interaction models, and associated developments. Environ. Plan. A 1971, 3 , 1-32. [CrossRef]

25. Duenas, M.; Fagiolo, G. Modeling the international-trade network: A gravity approach. J. Econ. Interact. Coord. 2013, 8, 155-178. [CrossRef]

26. Squartini, T.; Garlaschelli, D. Jan Tinbergen's legacy for economic networks: From the gravity model to quantum statistics. In Econophysics of Agent-Based Models; Abergel, F., Aoyama, H., Chakrabarti, B., Chakraborti, A., Ghosh, A., Eds.; Springer: Chambridge, UK, 2013; pp. 161-186, ISBN 978-3-319-00022-0.

27. Squartini, T.; Picciolo, F.; Ruzzenenti, F.; Garlaschelli, D. Reciprocity of weighted networks. Sci. Rep. 2013, 3, 2729. [CrossRef] [PubMed]

28. Chiarucci, R.; Ruzzenenti, F.; Loffredo, M.I. Detecting spatial homogeneity in the world trade web with Detrended Fluctuation Analysis. Phys. A Stat. Mech. Its Appl. 2014, 401, 1-7. [CrossRef]

29. Buch, C.M.; Kleinert, J.; Toubal, F. The distance puzzle: On the interpretation of the distance coefficient in gravity equations. Econ. Lett. 2004, 83, 293-298. [CrossRef]

30. Cairncross, F. The Death of Distance: How the Communications Revolution Will Change Our Lives; Harvard Business School Press: Cambridge, MA, USA, 1997.

31. Lin, F. Are distance effects really a puzzle? Econ. Model. 2013, 31, 684-689. [CrossRef]

32. Karpiarz, M.; Fronczak, P.; Fronczak, A. International trade network: Fractal properties and globalization puzzle. Phys. Rev. Lett. 2014, 113, 248701. [CrossRef] [PubMed]

33. Masucci, A.P.; Serras, J.; Johansson, A.; Batty, M. Gravity versus radiation models: On the importance of scale and heterogeneity in commuting flows. Phys. Rev. E 2013, 88, 022812. [CrossRef] [PubMed]

34. Ruzzenenti, F.; Garlaschelli, D.; Basosi, R. Complex networks and symmetry II: Reciprocity and evolution of world trade. Symmetry 2010, 2, 1710-1744. [CrossRef]

35. Haldane, A.G.; May, R.M. Systemic risk in banking ecosystems. Nature 2011, 469, 351-355. [CrossRef] [PubMed]

36. Garlaschelli, D.; Loffredo, M.I. Fitness-dependent topological properties of the world trade web. Phys. Rev. Lett. 2004, 93, 188701. [CrossRef] [PubMed] 
37. Fagiolo, G.; Reyes, J.; Schiavo, S. The evolution of the world trade web: A weighted-network analysis. J. Evolut. Econ. 2010, 20, 479-514. [CrossRef]

38. Ruzzenenti, F.; Basosi, R. Modelling the rebound effect with network theory: An insight into the European freight transport sector. Energy 2017, 118, 272-283. [CrossRef]

39. Wang, J.; Mo, H.; Wang, F.; Jin, F. Exploring the network structure and nodal centrality of China's air transport network: A complex network approach. J. Transp. Geogr. 2011, 19, 712-721. [CrossRef]

40. Ducruet, C.; Beauguitte, L. Spatial science and network science: Review and outcomes of a complex relationship. Netw. Spat. Econ. 2014, 14, 297-316. [CrossRef]

41. Rocha, L.E. Dynamics of air transport networks: A review from a complex systems perspective. Chin. J. Aeronaut. 2017, 30, 469-478. [CrossRef]

42. Calatayud, A.; Mangan, J.; Palacin, R. Connectivity to international markets: A multi-layered network approach. J. Transp. Geogr. 2017, 61, 61-71. [CrossRef]

43. Surana, A.; Kumara, S.; Greaves, M.; Raghavan, U.N. Supply-chain networks: A complex adaptive systems perspective. Int. J. Prod. Res. 2005, 43, 4235-4265. [CrossRef]

44. Pathak, S.D.; Day, J.M.; Nair, A.; Sawaya, W.J.; Kristal, M.M. Complexity and adaptivity in supply networks: Building supply network theory using a complex adaptive systems perspective. Decis. Sci. 2013, 38, 547-580. [CrossRef]

45. Hearnshaw, E.J.; Wilson, M.M. A complex network approach to supply chain network theory. Int. J. Oper. Prod. Manag. 2013, 33, 442-469. [CrossRef]

46. Bellamy, M.A.; Basole, R.C. Network analysis of supply chain systems: A systematic review and future research. Syst. Eng. 2013, 16, 235-249. [CrossRef]

47. Ruzzenenti, F.; Picciolo, F.; Basosi, R.; Garlaschelli, D. Spatial effects in real networks: Measures, null models, and applications. Phys. Rev. E 2012, 86, 066110. [CrossRef] [PubMed]

48. Squartini, T.; Picciolo, F.; Ruzzenenti, F.; Basosi, R.; Garlaschelli, D. Disentangling spatial and non-spatial effects in real networks. In Complex Networks and their Applications; Cherifi, H., Ed.; Cambridge Scholars Publishing: Newcastle upon Tyne, UK, 2014; pp. 1-28, ISBN 978-1-4438-5370-5.

49. Squartini, T.; Garlaschelli, D. Analytical maximum-likelihood method to detect patterns in real networks. New J. Phys. 2011, 13, 083001. [CrossRef]

50. Ruzzenenti, F.; Basosi, R. Evaluation of the energy efficiency evolution in the European road freight transport sector. Energy Policy 2009, 37, 4079-4085. [CrossRef] 